\title{
Photometric and Coravel observations of red giant candidates in three open clusters: membership, binarity, reddening and metallicity
}

\author{
J. J. Clariá ${ }^{1}$, J.-C. Mermilliod ${ }^{2}$, A. E. Piatti ${ }^{3}$, and M. C. Parisi ${ }^{1}$ \\ 1 Observatorio Astronómico, Laprida 854, 5000, Córdoba, Argentina \\ e-mail: claria@oac.uncor.edu \\ 2 Laboratoire d'Astrophysique de l'École polytechnique fédérale de Lausanne, Observatoire, 1290 Sauverny, Switzerland \\ 3 Instituto de Astronomía y Física del Espacio, CC 67, Suc. 28, 1428 Buenos Aires, Argentina
}

Received 19 December 2005 / Accepted 16 February 2006

\begin{abstract}
Aims. We present new CORAVEL radial-velocity observations and photoelectric photometry in the $U B V$ and $D D O$ systems for a sample of potential members of the red-giant branches of NGC 6192, NGC 6208 and NGC 6268, three open clusters projected close to the Galactic center direction. We also examine the properties of a sample of 42 inner disk open clusters projected towards almost the same direction as the three clusters.

Methods. Cluster members and red field giants were discriminated by using the CORAVEL radial-velocity data and by applying two photometric criteria. Interstellar reddening and metal content of the clusters were derived from combined BV and DDO data. Results. Cluster membership for five red giants in NGC 6192, three in NGC 6208 and three in NGC 6268 has been confirmed by the analysis of the photometric and kinematic data. Photometric membership probabilities show very good agreement with those obtained from CORAVEL radial velocities. Three new spectroscopic binaries were discovered among the red giants of NGC 6192 and NGC 6208. Mean radial velocities and $E(B-V)$ colour excesses were derived.

Conclusions. The overall metallicities were found to be nearly solar for NGC 6208 and above solar for NGC 6192 and NGC 6268. Most of the clusters located closer than $2 \mathrm{kpc}$ from the Sun in the considered direction are slightly more reddened than the absorption resulting from the Baade's window absorption law.
\end{abstract}

Key words. Galaxy: open clusters and associations: general - stars: binaries: spectroscopic - techniques: photometric techniques: radial velocities - stars: abundances

\section{Introduction}

In this paper we discuss NGC 6192, NGC 6208 and NGC 6268 as part of a more extensive study of red giants in open clusters using CORAVEL radial velocity data and new photoelectric photometry. These three poorly studied, southern, open clusters are located near the Galactic plane and close to the Galactic center direction. Their corresponding equatorial and Galactic coordinates are given in Table 1, together with the Trumpler (1930) class according to Archinal \& Hynes (2003), the $E(B-V)$ colour excesses, distances from the Sun and ages given by the WEBDA open Cluster Database (Mermilliod \& Paunzen 2003). These clusters are interesting in themselves because of the number of red giant candidates they contain. Neither radial-velocity determination nor $D D O$ photometry for any of the stars of these three clusters has been published yet.

NGC 6192 (C1636-432) - also known as Mel 109 (Melotte 1915), Cr 309 (Collinder 1931) or BH 194 (van den Bergh \& Hagen 1975) - has been the most thoroughly studied of our three targets. However, a remarkable disagreement about the cluster reddening value is found in the literature and, consequently, discrepancies in ages and distances have been reported. Kilambi \& Fitzgerald (1983, hereafter KF83) and King (1987) derived a reddening of $E(B-V)=0.26$, a rather old age
( $1.0 \mathrm{Gyr})$ and a distance from the Sun of about $1 \mathrm{kpc}$, based on photographic and $C C D$ photometry, respectively. More recently, Kjeldsen \& Frandsen (1991, hereafter KF91) obtained a much higher reddening of $E(B-V)=0.68$, an age of scarcely $89 \mathrm{Myr}$ and a distance of about $1.7 \mathrm{kpc}$. Paunzen et al. (2003) obtained CCD photometry in the uvby Strömgren's system to clarify the abovementioned discrepancies. They derived $[\mathrm{Fe} / \mathrm{H}]=-0.10 \pm$ 0.09 and $E(b-y)=0.40$, equivalent to $E(B-V)=0.54$, if the relation $E(B-V)=1.35 E(b-y)$ given by Crawford (1978) is used. This reddening value seems to support KF91's estimate and apparently contradicts the values previously reported by KF83 and King (1987). Loktin et al. (2001) published a second version of their Open Cluster Catalogue (Loktin \& Matkin 1994) reviewing the fundamental parameters of 423 clusters. Their updated distances are now based on a Hyades distance modulus of 3.27, this value being 0.06 mag lower than that found from Hipparcos parallaxes, namely $V-M_{V}=3.33 \pm 0.01$ (Perryman et al. 1998). By using the original data from KF91, Loktin et al. (2001) determined the following parameters for NGC 6192: $E(B-V)=$ $0.637, d=1547 \mathrm{pc}$ and $\log t=8.13$ (Table 1).

NGC 6208 (C1645-537) - also designated $\mathrm{Cr} 313$ or BH 198 - is located $\sim 26^{\circ}$ from the Galactic center direction in a very rich star field in Ara, which makes its membership and astrophysical properties rather difficult to investigate. 
Table 1. Open clusters studied.

\begin{tabular}{lcccccccc}
\hline \hline Cluster & $\begin{array}{c}\alpha(\mathrm{J} 2000) \\
(\mathrm{h}: \mathrm{m})\end{array}$ & $\begin{array}{c}\delta(\mathrm{J} 2000) \\
\left({ }^{\circ}:^{\prime}\right)\end{array}$ & $\begin{array}{c}l \\
\left({ }^{\circ}\right)\end{array}$ & $\begin{array}{c}b \\
\left({ }^{\circ}\right)\end{array}$ & Trumpler class & $\begin{array}{c}E(B-V) \\
(\mathrm{mag})\end{array}$ & $\begin{array}{c}d \\
(\mathrm{kpc})\end{array}$ & $\log t$ \\
\hline NGC 6192 & 1640.4 & -4322.0 & 340.7 & 2.1 & I2r & 0.637 & 1.5 & 8.13 \\
NGC 6208 & 1649.5 & -5343.0 & 333.8 & -5.8 & III2r & 0.210 & 0.9 & 9.07 \\
NGC 6268 & 1702.7 & -3944.3 & 346.1 & 1.2 & II2p & 0.387 & 1.0 & $8.40^{a}$ \\
\hline
\end{tabular}

${ }^{a}$ The age given by WEBDA is incorrect (see Sect. 1).

To the best of our knowledge, the only photometric study was performed by Lindoff (1972), who obtained $U B V$ photoelectric and photographic photometry for 17 and 286 stars, respectively, in the cluster field. Although he derived $E(B-V)=$ 0.18 , an age of $\sim 1 \mathrm{Gyr}$ and a distance of about $1 \mathrm{kpc}$ from the Sun, caution should be exercised when analyzing these results owing to the huge dispersion existing in the Lindoff $(V, B-V)$ colour-magnitude diagram (CMD, see Sect. 3.1). Using Lindoff's data, Loktin et al. (2001) obtained similar basic parameters for this cluster (Table 1). On the other hand, based on unpublished $D D O$ photometric data of 3 red giant candidates, Piatti et al. (1995) obtained $[\mathrm{Fe} / \mathrm{H}]=-0.03$, while Twarog et al. (1997) revised this value to $[\mathrm{Fe} / \mathrm{H}]=0.00$. More recently, Paunzen \& Maitzen (2001) performed a $C C D$ photometric search for chemically peculiar (CP) stars in NGC 6208 but no apparent peculiar object was found.

NGC 6268 (C1658-396) - also known as Cr 323 or BH 212 is located at less than $14^{\circ}$ from the Galactic center in Scorpius. As far as we know, the only photometric $U B V$ study was done by Seggewiss (1968), based on photographic plates. Using the original photographic data obtained by Seggewiss, Loktin et al. (2001) derived $E(B-V)=0.387$, a distance of $1029 \mathrm{pc}$ from the Sun and an age of scarcely $17 \mathrm{Myr}(\log t=7.25)$. As shown in Fig. 5, however, this age has been misestimated. So much so that adopting the theoretical metal content $Z=0.019$ and using the above mentioned reddening and distance, the Padova isochrone (Girardi et al. 2000) of $\log t=8.35(t=224 \mathrm{Myr})$ most accurately reproduces the cluster features in the $(V, B-V)$ CMD. Recently, McSwain \& Gies (2005) identified one definite Be star in this cluster from observations performed with the Strömgren $b$ and $y$ and the narrow band $\mathrm{H}_{\alpha}$ filters, while Paunzen et al. (2005) detected several CP stars using the narrow band, three filter $\Delta \alpha$ photometric system. By fitting theoretical isochrones for the $\Delta \alpha$ system (Claret et al. 2003), Paunzen et al. (2005) derived $E(B-V)=0.40$, a distance of $1080 \mathrm{pc}$ from the Sun and ane of $40 \mathrm{Myr}(\log t=7.60)$.

As part of a long-term project to determine abundances and astrophysical properties of red evolved stars in southern open clusters, we present here CORAVEL radial velocities and accurate $U B V$ and $D D O$ photometry of $\mathrm{G}$ and/or $\mathrm{K}$ giant candidates in the field of the abovementioned clusters. These data are used to discuss cluster membership, to detect new spectroscopic binaries, to make an independent estimate of the individual $E(B-V)$ colour excesses, as well as to derive the metal content of the clusters.

In Sect. 2, we briefly describe the observational material. Section 3 presents the analysis and discussion of the photometric and CORAVEL data. Using the WEBDA Open Cluster Database (Mermilliod \& Paunzen 2003), we examine in Sect. 4 the properties of a sample of open clusters located almost in the same direction as NGC 6192, NGC 6208 and NGC 6268. A short summary of our main conclusions is given in Sect. 5 .

\section{The observational material}

\subsection{Photometric UBV and DDO observations}

Thirty seven potential members of the red-giant branches of NGC 6192, NGC 6208 and NGC 6268 were originally selected from their existing CMDs. These stars appear to be free from contamination by neighbouring stars and were observed in the $U B V$ system, while only 22 of them were observed in the $D D O$ photometric system (McClure 1976). Only the four primary filters of the $D D O(41,42,45$ and 48) system were used because they provide adequate information for the present purposes.

The $U B V$ and $D D O$ measurements were made with the 1.0-m telescopes of the Cerro Tololo Inter-American Observatory (CTIO) and La Silla (LS) Observatory, located in Chile, in April 1994 and June 1993, respectively. Single-channel pulse-counting photometers were used in both observatories in conjunction with dry-ice refrigerated Hamamatsu phototubes. Mean extinction coefficients for CTIO and LS were used, and between 11 and 18 standard stars from the lists of Cousins (1973, 1974), Graham (1982) and McClure (1976) were observed nightly to place the observations in the standard $U B V$ and $D D O$ systems. The external and internal mean errors of the $U B V$ and $D D O$ photometries are similar to those quoted in previous papers (e.g. Clariá et al. 2005). Some $U B V$ measurements were also made in March 1988 with the LS 1.0-m telescope and a RCA 31034 (Quantacom) Ga-As photomultiplier. No evidence was found of systematic discrepancies either in $V$ or in the colours among the stars observed in common at LS and CTIO. Therefore, mean values were used and are listed in Table 2, together with their mean internal errors $\sigma$, in units of $0.001 \mathrm{mag}$, and the number $n_{1}$ on which each star was observed in the $U B V$ system. Star designations are from KF83, Lindoff (1972) and Seggewiss (1967) for NGC 6192, NGC 6208 and NGC 6268, respectively, except for stars 255, 265 and 274 of NGC 6192, which are identified in Fig. 1.

Table 3 presents the observed $D D O$ colours, along with their mean internal errors $\sigma$, in units of $0.001 \mathrm{mag}$, and the number $n_{2}$ on which each star was observed in this system.

\subsection{Comparison with other UBV data}

The agreement between the present $U B V$ data for the stars measured in NGC 6192 and those obtained photographically by KF83 is very poor. The mean differences $(\Delta=$ KF83 minus present value) and standard deviations from 8 stars measured in common are: $\Delta V=-0.02 \pm 0.14, \Delta(B-V)=-0.34 \pm 0.10$ and $\Delta(U-B)=0.13 \pm 0.25$. These large differences, particularly in the $B-V$ colours, are almost entirely due to the low accuracy of KF83's photographic data. Moreover, they seem to explain the abovementioned wide discrepancies found in the reddening values derived by other authors. There are only two stars 
Table 2. $U B V$ photometry of red giant candidates.

\begin{tabular}{rccccccc}
\hline \hline Star & $V$ & $\sigma$ & $B-V$ & $\sigma$ & $U-B$ & $\sigma$ & $n_{1}$ \\
\hline NGC 6192 & & & & & & & \\
4 & 11.588 & 6 & 1.334 & 15 & 1.161 & 4 & 3 \\
9 & 11.430 & 8 & 1.510 & 16 & 1.876 & 34 & 2 \\
28 & 10.663 & 8 & 1.991 & 8 & 2.339 & 24 & 2 \\
45 & 11.743 & 3 & 1.546 & 13 & 1.481 & 33 & 2 \\
91 & 11.207 & 1 & 1.632 & 10 & 1.388 & 11 & 2 \\
96 & 11.306 & 6 & 1.502 & 12 & 1.341 & 20 & 2 \\
$137^{a}$ & 11.223 & 7 & 1.758 & 8 & 1.633 & 4 & 3 \\
253 & 11.130 & 9 & 1.111 & 10 & 0.749 & 10 & 2 \\
$255^{b}$ & 11.945 & 10 & 1.597 & 16 & 1.363 & 11 & 3 \\
$265^{b}$ & 11.849 & 29 & 1.451 & 3 & 1.323 & 10 & 2 \\
$274^{b}$ & 11.300 & 13 & 1.650 & 13 & 1.512 & 13 & 3 \\
NGC 6208 & & & & & & & \\
9 & 12.563 & 12 & 1.386 & 11 & 1.405 & 45 & 2 \\
$19^{a}$ & 11.111 & 26 & 1.308 & 19 & 1.045 & 16 & 3 \\
$20^{a}$ & 12.449 & 14 & 1.394 & 19 & 1.316 & 31 & 3 \\
27 & 11.151 & 7 & 1.265 & 4 & 0.873 & 1 & 2 \\
31 & 11.643 & 10 & 1.203 & 17 & 0.882 & 9 & 3 \\
$44^{c}$ & 12.907 & 18 & 0.958 & 8 & 0.347 & 33 & 3 \\
48 & 11.632 & 19 & 1.325 & 17 & 1.098 & 11 & 3 \\
119 & 12.616 & 10 & 1.815 & 21 & 2.124 & 80 & 5 \\
124 & 12.309 & 22 & 1.363 & 5 & 1.232 & 12 & 3 \\
$127^{c}$ & 12.927 & 28 & 1.469 & 23 & 1.377 & 84 & 3 \\
132 & 14.586 & 15 & 2.169 & 53 & & & 2 \\
133 & 11.575 & 1 & 1.331 & 2 & 1.328 & 22 & 2 \\
150 & 12.461 & 19 & 1.375 & 7 & 1.152 & 15 & 2 \\
$165^{a}$ & 12.804 & 13 & 1.707 & 15 & 1.804 & 65 & 2 \\
166 & 12.158 & 16 & 1.253 & 2 & 1.023 & 30 & 2 \\
$178^{c}$ & 13.093 & 12 & 1.375 & 8 & 1.169 & 78 & 3 \\
179 & 12.987 & 15 & 1.471 & 12 & 1.535 & 63 & 3 \\
209 & 12.575 & 9 & 0.644 & 12 & 0.125 & 16 & 3 \\
267 & 12.550 & 29 & 1.646 & 24 & 1.716 & 70 & 2 \\
NGC 6268 & & & & & & & \\
13 & 10.415 & 5 & 1.242 & 3 & 0.979 & 13 & 2 \\
22 & 10.811 & 27 & 1.435 & 2 & 1.181 & 15 & 2 \\
35 & 10.349 & 7 & 1.685 & 23 & 1.669 & 3 & 2 \\
44 & 9.965 & 25 & 1.395 & 9 & 1.137 & 5 & 2 \\
48 & 11.311 & 17 & 1,218 & 5 & 1.016 & 5 & 2 \\
73 & 11.189 & 23 & 1.465 & 7 & 1.380 & 1 & 2 \\
$76^{c}$ & 9.749 & 15 & 1.394 & 15 & 1.075 & 15 & 2 \\
\hline & & & & & & &
\end{tabular}

${ }^{a}$ Double star, only the bright component was measured.

${ }^{b}$ Star identified in Fig. 1.

${ }^{c}$ Double star, both components were measured together.

photoelectrically measured by both KF83 and by this study: Nos. 96 and 137. However, only the data for star 96 can be compared, since star 137 is a binary system for which only the brightest component was measured. The corresponding differences in $V, B-V$ and $U-B$ for star 96 are $-0.02,-0.02$ and -0.20 , respectively.

Among the stars measured in NGC 6268, four (Nos. 19, 27, 31 and 48) were also observed photoelectrically by Lindoff (1972) in the $U B V$ system. Their $V$ magnitudes show a rather poor agreement with the present ones, while the $B-V$ and $U-B$ colours exhibit a relatively good agreement. The mean differences (Lindoff minus present values) are: $\Delta V=-0.08 \pm 0.09$, $\Delta(B-V)=-0.03 \pm 0.03$ and $\Delta(U-B)=-0.03 \pm 0.10$. Regarding NGC 6268, the mean differences for the stars measured in common between Seggewiss's (1967) photographic values and our own values are: $\Delta V=0.06 \pm 0.06, \Delta(B-V)=-0.01 \pm 0.08$ and $\Delta(U-B)=-0.25 \pm 0.09$.

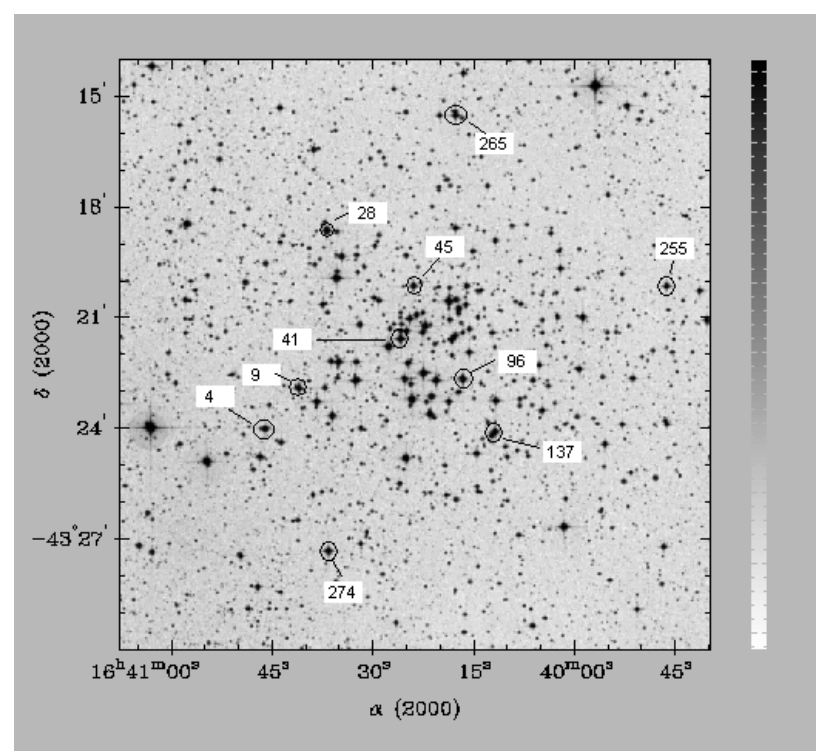

Fig. 1. $R$ image of NGC 6192 from the Digitized Sky Survey (DSS). Stars 255, 265 and 274 are identified. North is up and east is to the left.

Table 3. $D D O$ photometry of red giant candidates.

\begin{tabular}{rrrrrrrr}
\hline \hline Star & $C 4548$ & $\sigma$ & $C 4245$ & $\sigma$ & $C 4142$ & $\sigma$ & $n_{2}$ \\
\hline NGC 6192 & & & & & & & \\
4 & 1.301 & 10 & 1.334 & 12 & 0.233 & 11 & 2 \\
9 & 1.343 & 22 & 0.931 & 9 & 0.293 & 7 & 2 \\
28 & 1.563 & 11 & 1.320 & 8 & 0.302 & 12 & 2 \\
45 & 1.392 & 17 & 0.921 & 10 & 0.309 & 4 & 2 \\
91 & 1.432 & 11 & 0.990 & 16 & 0.359 & 4 & 2 \\
96 & 1.351 & 22 & 0.851 & 17 & 0.338 & 15 & 2 \\
$137^{a}$ & 1.483 & 8 & 1.057 & 12 & 0.430 & 1 & 2 \\
253 & 1.196 & 10 & 0.807 & 10 & 0.223 & 14 & 2 \\
255 & 1.353 & 8 & 1.003 & 11 & 0.213 & 12 & 2 \\
265 & 1.300 & 6 & 1.048 & 9 & 0.145 & 10 & 2 \\
274 & 1.424 & 12 & 1.056 & 10 & 0.247 & 8 & 2 \\
NGC 6208 & & & & & & & \\
$19^{a}$ & 1.279 & 6 & 0.935 & 11 & 0.233 & 3 & 2 \\
27 & 1.261 & 3 & 0.853 & 4 & 0.171 & 27 & 2 \\
31 & 1.250 & 8 & 0.851 & 4 & 0.177 & 3 & 2 \\
48 & 1.266 & 8 & 0.953 & 1 & 0.200 & 7 & 2 \\
133 & 1.288 & 4 & 1.007 & 21 & 0.308 & 1 & 2 \\
NGC 6268 & & & & & & & \\
13 & 1.235 & 12 & 0.887 & 16 & 0.227 & 15 & 2 \\
22 & 1.318 & 11 & 0.924 & 14 & 0.303 & 3 & 2 \\
44 & 1.330 & 12 & 0.932 & 1 & 0.333 & 8 & 2 \\
48 & 1.206 & 11 & 0.937 & 6 & 0.240 & 16 & 2 \\
73 & 1.365 & 13 & 0.976 & 11 & 0.353 & 17 & 2 \\
$76^{b}$ & 1.312 & 6 & 0.904 & 12 & 0.248 & 5 & 2 \\
\hline
\end{tabular}

${ }^{a}$ Double star, only the bright component was measured.

${ }^{b}$ Double star, both components were measured together.

\subsection{CORAVEL radial velocities}

High precision radial velocity observations have been obtained for the program stars between June 1984 and Septembre 1996 as part of the regular observing campaign on red giants in open clusters, using the photoelectric scanner CORAVEL (Baranne et al. 1979) installed on the 1.54-m Danish telescope at LS (Chile). The radial velocities are in the system defined by Udry et al. (1999), and calibrated with high-precision data from the ELODIE spectrograph (Baranne et al. 1996). A total of 82 observations were obtained for 24 red giant candidates in 
Table 4. CORAVEL radial-velocity data.

\begin{tabular}{rrcccccl}
\hline \hline Star & \multicolumn{1}{c}{$V_{r}$} & $\epsilon$ & $\sigma$ & $E / I$ & $P\left(\chi^{2}\right)$ & $N$ & Notes \\
\hline NGC 6192 & & & & & & & \\
4 & +45.28 & 0.53 & 0.76 & 1.60 & 0.110 & 2 & $\mathrm{NM}$ \\
9 & -8.16 & 0.22 & 0.45 & 1.01 & 0.391 & 4 & $\mathrm{M}$ \\
28 & +24.81 & 0.39 & 0.55 & 1.35 & 0.179 & 2 & $\mathrm{NM}$ \\
45 & -8.06 & 0.23 & 0.43 & 0.96 & 0.435 & 4 & $\mathrm{M}$ \\
91 & -8.82 & 0.59 & 1.57 & 3.69 & 0.000 & 7 & $\mathrm{M}, \mathrm{SB}$ \\
96 & -6.40 & 0.59 & 1.87 & 3.87 & 0.000 & 10 & $\mathrm{M}, \mathrm{SB}$ \\
137 & -7.17 & 0.33 & 0.65 & 1.54 & 0.071 & 4 & $\mathrm{M}$ \\
253 & -19.32 & 0.26 & 0.45 & 1.00 & 0.365 & 3 & $\mathrm{NM}$ \\
255 & -88.51 & 0.34 & 0.19 & 0.40 & 0.687 & 2 & $\mathrm{NM}$ \\
265 & -59.69 & 0.33 & 0.26 & 0.57 & 0.571 & 2 & $\mathrm{NM}$ \\
NGC 6208 & & & & & & & \\
9 & -26.29 & 0.31 & 0.21 & 0.49 & 0.627 & 2 & $\mathrm{NM}$ \\
19 & -32.17 & 0.21 & 0.12 & 0.34 & 0.889 & 3 & $\mathrm{M}$ \\
20 & +9.96 & 0.45 & 0.45 & & & 1 & $\mathrm{NM}$ \\
27 & -69.01 & 2.26 & 3.19 & 2.27 & 0.023 & 2 & $\mathrm{NM}$ \\
31 & -32.83 & 0.23 & 0.11 & 0.28 & 0.923 & 3 & $\mathrm{M}$ \\
48 & -31.63 & 2.30 & 6.89 & 17.14 & 0.000 & 9 & $\mathrm{M}, \mathrm{SB}$ \\
124 & -49.12 & 0.41 & 0.41 & & & 1 & $\mathrm{NM}$ \\
133 & +29.30 & 0.37 & 0.37 & & & 1 & $\mathrm{NM}$ \\
150 & -113.92 & 0.46 & 0.46 & & & 1 & $\mathrm{NM}$ \\
166 & -43.48 & 0.40 & 0.40 & & & 1 & $\mathrm{NM}$ \\
13 & -2.59 & 0.19 & 0.30 & 0.77 & 0.623 & 4 & $\mathrm{NM}$ \\
44 & -15.28 & 0.23 & 0.38 & 0.82 & 0.581 & 4 & $\mathrm{M}$ \\
$76 \mathrm{~A}$ & -14.97 & 0.23 & 0.12 & 0.31 & 0.907 & 3 & $\mathrm{M}, \mathrm{VB}$ \\
$76 \mathrm{~B}$ & -15.09 & 0.28 & 0.43 & 0.87 & 0.466 & 3 & $\mathrm{M}, \mathrm{VB}$ \\
\hline NGC 6268 & & & & & & &
\end{tabular}

the three clusters, with an average of 3 observations per star. Columns (2) to (8) of Table 4 present in succession the mean radial velocity and its uncertainty in $\left[\mathrm{km} \mathrm{s}^{-1}\right]$, the dispersion in the mean radial velocities, the ratio of the external to internal errors $(E / I)$, the number of radial-velocity observations, the probability $\mathrm{P}\left(\chi^{2}\right)$ that the scatter is due to random noise and remarks on membership and duplicity. Individual observations, including the Julian date, are available from the WEBDA database (http://www.univie.ac.at/webda/) and upon request to the authors. All the stars in NGC 6192 were observed both photoelectrically and with the CORAVEL, while nine stars in NGC 6208 and one star in NGC 6268 were observed photoelectrically but not with the CORAVEL.

\section{Analysis and discussion of the data}

\subsection{Cluster membership and interstellar reddening}

The separation of red giant cluster members from the field stars was carried out primarily on the basis of the radial velocity data. As shown in Table 4, five stars of NGC 6192 (Nos. 9, 45, 91, 96 and 137) have CORAVEL radial velocities in the narrow range $-8.8 \mathrm{~km} \mathrm{~s}^{-1}<V_{r}<-6.4 \mathrm{~km} \mathrm{~s}^{-1}$, including two new spectroscopic binaries (SBs). All these stars appear to be unambiguously red giant members of NGC 6192. On the other hand, only three stars of NGC 6208 (Nos. 19, 31 and 48) - including one new spectroscopic binary - and three stars of NGC 6268 (Nos. 44, 76A and 76B) show nearly similar radial velocities. Therefore, their physical membership to NGC6208 and NGC 6268, respectively, appears to be highly probable. Mean radial velocities of $(-7.7 \pm 0.38) \mathrm{km} \mathrm{s}^{-1},(-32.21 \pm 0.28) \mathrm{km} \mathrm{s}^{-1}$ and $(-15.11 \pm 0.08) \mathrm{km} \mathrm{s}^{-1}$ are here derived for NGC 6192, NGC 6208 and NGC 6268, respectively. To derive these values, the radial velocities of the new SBs were also considered.

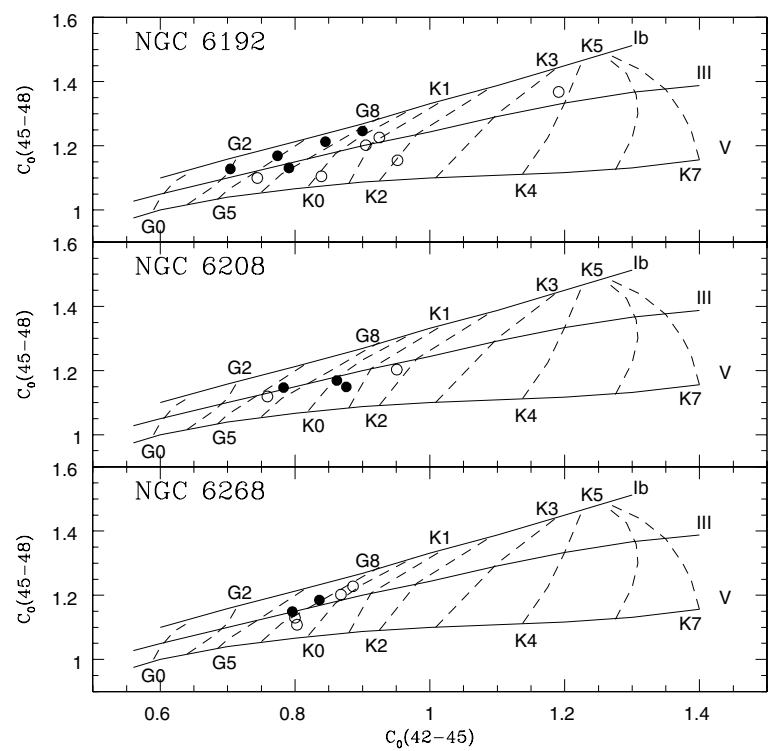

Fig. 2. Red giants in NGC 6192, NGC 6208 and NGC 6268 plotted on the unreddened $\left[C_{0}(45-48), C_{0}(42-45)\right]$ diagram. Solid lines are solar neighbourhood lines for luminosity classes Ib, III and V as defined by Clariá et al. (1994). Cluster members and field stars are represented by filled and open circles, respectively.

Clariá \& Lapasset (1983) and Clariá (1985) have shown that the likelihood of membership of a $\mathrm{G}$ or $\mathrm{K}$ star located in or near an open cluster can be evaluated with fairly good certainty by using two independent criteria - denoted A and B - based on combined $B V$ and $D D O$ photometric data. To apply criteria $\mathrm{A}$ and $\mathrm{B}$, we adopted the colour excesses for the main sequence stars and true distance moduli derived by Loktin et al. (2001) for the three clusters. The $D D O$ colours were dereddened according to the reddening coefficients given by McClure (1973) and the predicted luminosity class (LC) for each cluster star was determined from the Straiz̃ys (1992) calibration assuming $R=A_{V} / E(B-V)=3.5$ for G-K III stars (Straiz̃ys 1992). Figure 2 illustrates the cluster giant branches in the unreddened $[\mathrm{Co}(45-48), \mathrm{Co}(42-45)]$ diagrams. Also included in this figure are the solar neighbourhood loci of Clariá et al. (1994) for luminosity classes Ib, III and V. Stars considered to be giant members and red field objects are represented by filled and open circles, respectively.

Table 5 presents the results of applying the photometric criteria. The successive columns contain the star identification, the colour excess $E(B-V)_{\mathrm{GK}}$ derived from Janes (1977) iterative procedure using $B V$ and $D D O$ data, the standard deviation of the $E(B-V)_{\mathrm{GK}}$ colour excess calculated from Eq. (2) of Clariá \& Lapasset (1983), the LC each star should have in order to be a cluster member, the spectral type derived from the DDO colours using the calibration of Clariá et al. (1994), the results from applying criteria A and B and the membership status finally assigned to each star. Star 96 of NGC 6192 falls slightly outside the range of Janes (1977) Table 1. If for this star we adopt the mean reddening derived from the other four cluster members, namely $E(B-V)=0.63$, then it turns out to be a cluster member according to criterion $\mathrm{B}$.

Although caution should be exercised when considering the results obtained from criterion A because of the probable nonuniform reddening in the cluster fields, there is good agreement between the photometric analysis and the kinematic data. With the exception of star 133 of NGC 6208 (photometric member 
Table 5. Photometric membership results.

\begin{tabular}{rcccllll}
\hline \hline Star & $C E$ & $\sigma_{\mathrm{CE}}$ & LC & $M K K$ & \multicolumn{2}{c}{ Criteria } & Mem \\
& $(\mathrm{mag})$ & $(\mathrm{mag})$ & $(\mathrm{Pred})$ & $(\mathrm{DDO})$ & $(\mathrm{A})$ & $(\mathrm{B})$ & \\
\hline NGC 6192 & & & & & & & \\
4 & 0.28 & 0.04 & II-III & K1 III & NM & $\mathrm{M}$ & $\mathrm{NM}$ \\
9 & 0.60 & 0.06 & II-III & G8 III & M & M & M \\
28 & 0.55 & 0.04 & II & K3/4 III & NM & PM & NM \\
45 & 0.63 & 0.05 & II-III & G5 II & M & M & M \\
91 & 0.62 & 0.04 & II-III & G5/8 II & M & M & M \\
96 & $0.63^{a}$ & - & II-III & G2 III & - & M & M \\
137 & 0.67 & 0.03 & II-III & G8/K0 III & M & M & M \\
253 & 0.27 & 0.04 & II-III & G5/8III-IV & NM & PM & NM \\
255 & 0.70 & 0.04 & II-III & K0IV-V & PM & NM & NM \\
265 & 0.41 & 0.02 & II-III & K2 IV & NM & NM & NM \\
274 & 0.56 & 0.04 & II-III & K1 III & NM & M & NM \\
NGC 6208 & & & & & & & \\
19 & 0.31 & 0.05 & III & K0/1 III-IV & PM & M & M \\
27 & 0.40 & 0.02 & III & G5 III & NM & M & NM \\
31 & 0.29 & 0.04 & III & G5/8 III & PM & M & M \\
48 & 0.33 & 0.06 & III & K0/1 III & PM & M & M \\
133 & 0.24 & 0.03 & III & K1/2 III & M & M & M \\
NGC 6268 & & & & & & & \\
13 & 0.36 & 0.04 & III & G9 IV-V & M & NM & NM \\
22 & 0.53 & 0.03 & III & G8/9 III-IV & NM & PM & NM \\
44 & 0.41 & 0.03 & III & G8 II-III & M & M & M \\
48 & 0.22 & 0.03 & III & K0/1 IV & NM & PM & NM \\
73 & 0.46 & 0.03 & III & K0 II-III & NM & M & NM \\
76 & 0.46 & 0.04 & III & G8 III & PM & M & M \\
\hline
\end{tabular}

${ }^{a}$ Outside the range of Janes (1977) calibration.

but CORAVEL non-member), all the stars considered to be cluster members according to the radial velocities, also are red giant members according to the photometric criteria. Therefore, as shown in previous papers (see, e.g. Clariá et al. 2003), criteria $\mathrm{A}$ and $\mathrm{B}$ lead to reliable membership results for $\mathrm{G}$ and $\mathrm{K}$ giants provided their $B V$ and $D D O$ photometric data are of high quality. The discrepancy found for star 133 of NGC 6208 may be due to the reddening adopted for the main sequence stars, i.e. $E(B-V)_{\mathrm{MS}}=0.21$ (Loktin et al. 2001). According to the individual $E(B-V)_{\mathrm{GK}}$ values obtained for the CORAVEL members of NGC 6208, the cluster reddening seems to be $\sim 0.1$ mag larger than that obtained by Loktin et al. (2001).

The mean $E(B-V)_{\mathrm{GK}}$ values derived here for the confirmed red giants of NGC 6192, NGC 6208 and NGC 6268 are $0.63 \pm$ $0.02,0.31 \pm 0.02$ and $0.43 \pm 0.02$, respectively. The present reddening values for NGC 6192 and NGC 6268 are in good agreement with those adopted for their main sequence stars, i.e. $E(B-V)_{\mathrm{MS}}=0.64$ and 0.39 , respectively. The present measurements specifically allow us to resolve the strong discrepancies existing in the literature regarding the reddening of NGC 6192. On the other hand, the reddening derived here for the red giants of NGC 6208 is clearly larger than that derived either by Lindoff (1972) or Loktin et al. (2001), namely 0.18 and 0.21, respectively. As shown in Fig. 4, if instead of 0.21 , a colour excess $E(B-V)=0.32$ is adopted for NGC 6208, the ischrone of $\log t=$ 9.10 fits very well the position of the cluster giants.

\subsection{Colour-magnitude diagrams}

The selection of the red star candidates for cluster membership was based on their positions in the CMDs. Therefore, the nonmember giants in NGC 6192 and NGC 6208 tend to be located in the same CMD region as the true members. This demonstrates

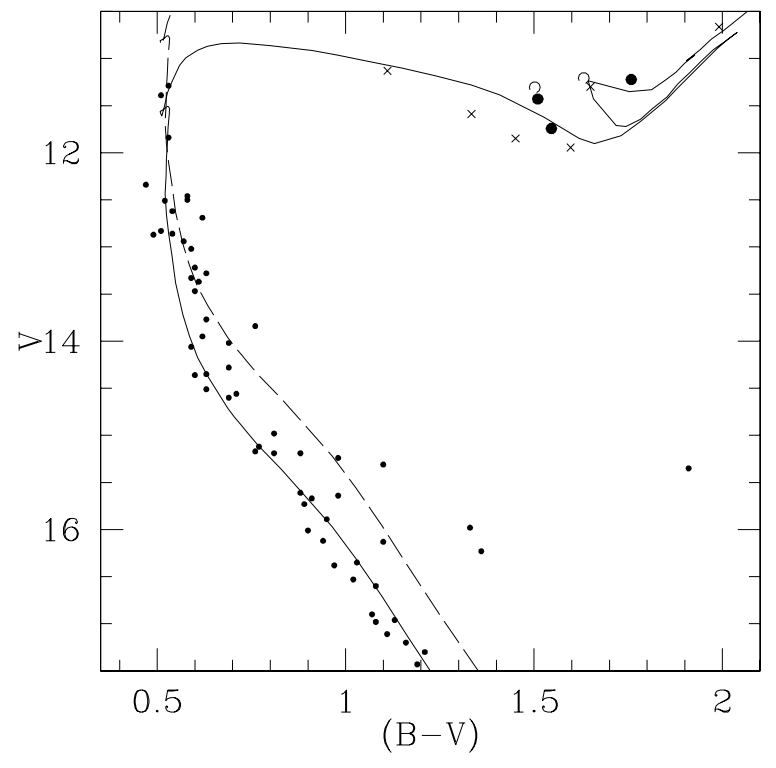

Fig. 3. The observed CMD for NGC 6192. The solar metallicity isochrone for $\log t=8.25$ from Girardi et al. (2000) has been adjusted to $E(B-V)=0.63$ and $V-M_{V}=13.00$ (Loktin et al. 2001). The dashed curve is the same isochrone shifted by 0.75 mag to reproduce the upper binary ridge. For the red giants, filled and open circles stand for single and binary members respectively, crosses, for non-members.

once again that radial velocity is a very strong membership criterion for red giants.

The fact that these red giants appear to be located at distances comparable to those of the clusters indicates that the clusters are embedded in a stellar field that has about the same age. This feature has been observed in other clusters, for example in Melotte 71 (Mermilliod et al. 1997) and in NGC 2324 and NGC 6259 (Mermilliod et al. 2001).

\subsubsection{NGC 6192}

To plot the main sequence in the CMD of NGC 6192 (Fig. 3) we have chosen the UBV CCD data of King (1987) after comparison of the various data sets. Given the value of $E(B-V)=$ 0.63 , the best fit is obtained with the isochrone for $\log t=8.25$, $Z=0.019$ of Girardi et al. (2000) and $m-M=13.00$. The dashed curve is the upper binary limit, i.e. the isochrone shifted by 0.75 mag.

Fitting an isochrone of higher metallicity is not possible for this value of the reddening. With an isochrone for $Z=0.030$, $E(B-V)=0.63, m-M=13.25$ and $\log t=8.20$, the red giant loop is much too red. The metallicity needed to reproduce the observed CM diagram in the red giant region should be intermediate between $Z=0.008$ and $Z=0.019$.

\subsubsection{NGC 6208}

Because there are no modern CCD data available for NGC 6208, we have computed $(B-V)$ indices from the $g 1-y$ indices of Paunzen \& Maitzen (2001) with the relation: $(B-V)=$ $2.508+2.772 *(g 1-y)$ (Paunzen 2006). The number of members appears to be small and the main sequence fit is not well constrained (Fig. 4). Given $E(B-V)=0.32$, one solution results in $m-M=11.15$ and $\log t=9.10$, with the isochrone of Girardi et al. (2000) for $Z=0.008$. It would be very useful to observe 


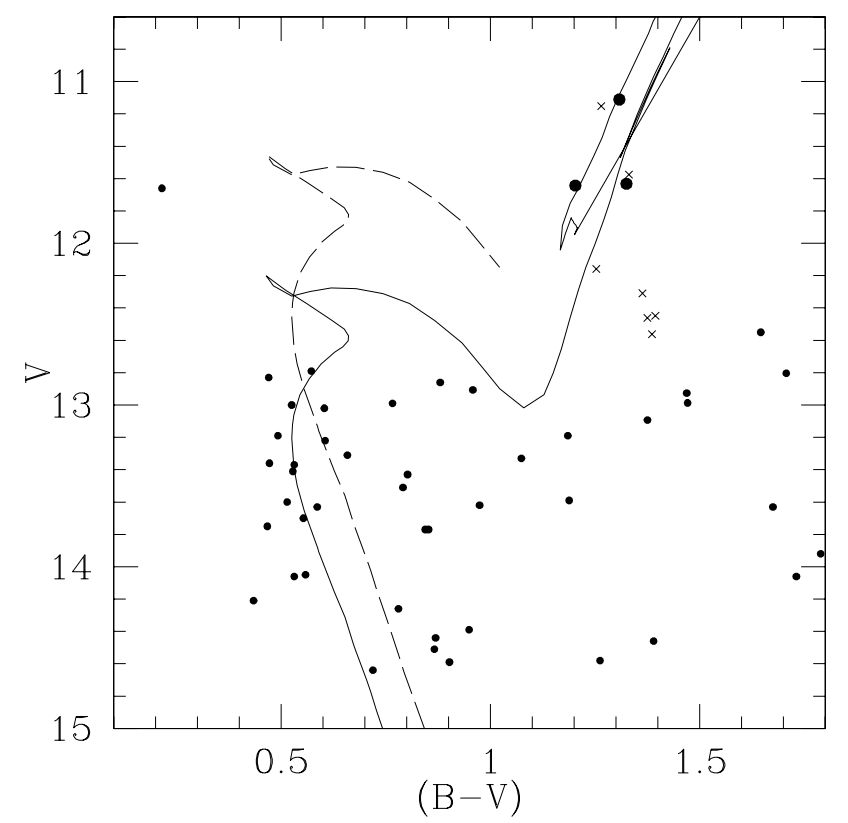

Fig. 4. The observed CMD for NGC 6208. The isochrone for $\log t=$ 9.10 and $Z=0.008$ from Girardi et al. (2000) has been adjusted to $E(B-V)=0.32$ and $V-M_{V}=11.15$. The dashed curve is the same isochrone shifted by $0.75 \mathrm{mag}$ to reproduce the upper binary ridge. Symbols are as in Fig. 3.

the field of NGC 6208 to identify the members and improve the parameter determination.

\subsubsection{NGC 6268}

In a similar manner, we have computed $(B-V)$ indices from the $(g 1-y)$ with the relation: $(B-V)=0.40+4.25(g 1-y)-$ $3.55(g 1-y)^{2}$ (Paunzen 2006). The resulting main sequence is rather well defined (Fig. 5). With the value of the colour excess determined from the red giants, $E(B-V)=0.38$, we obtained $m-M=11.25$ and $\log t=8.35$ for the isochrone of Girardi et al. (2000) for $Z=0.019$. These parameters are in good agreement with those published by Loktin et al. (2001).

With an isochrone of higher metallicity $(Z=0.030)$, it is not possible to fit simultaneously the main sequence and the red giants. The main sequence fit gives $E(B-V)=0.35, m-M=$ 11.35 and $\log t=8.40$, but the red giant loop is too red and too faint.

Note that star \#76 is a close visual binary formed by two red giants which have very similar radial-velocity values. This pair has not been numbered by Seggewiss (1967) and is located between stars \#28 and 30 on the Seggewiss (1967) map (see the scanned version on WEBDA). This cluster too would be a fruitful candidate for a modern CCD study.

\subsection{Metal content}

We have used the dereddened $D D O$ colour index $C_{0}(41-42)$ as an abundance indicator since it measures the strength of the $\lambda 4216$ cyanogen band, so that the larger the index, the greater the absorption by this band. Using this parameter, we have computed for each cluster giant the cyanogen anomaly, $\Delta C N$, defined by Piatti et al. (1993, hereafter PCM) as the difference between the dereddened $C_{0}(41-42)$ index of the observed star and the standard value of this index, which corresponds to a star

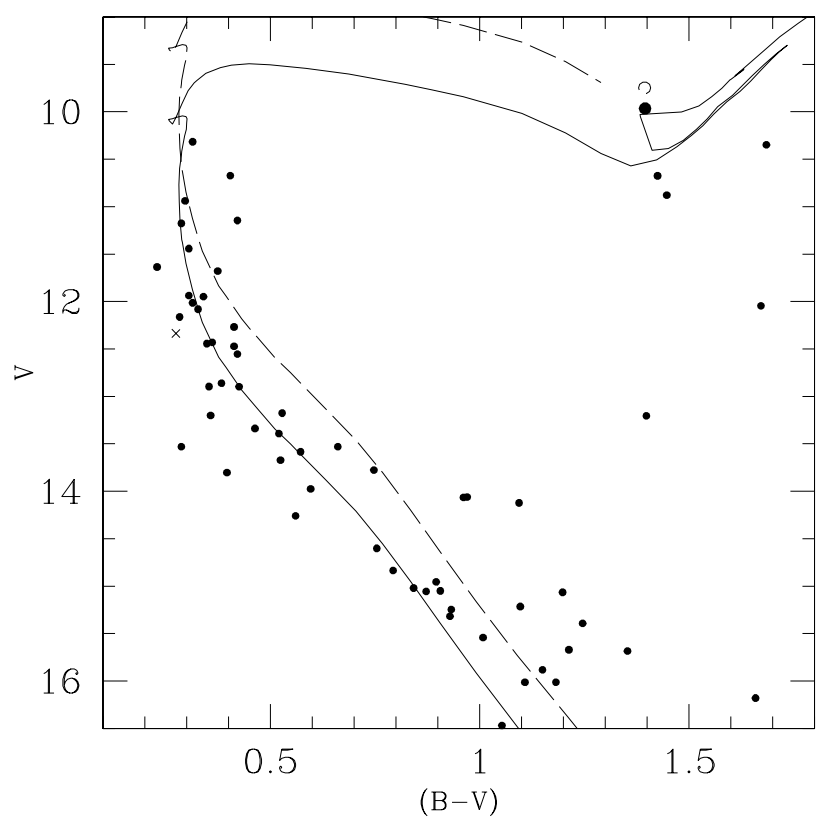

Fig. 5. The observed CMD for red evolved stars in NGC 6268. The solar metallicity isochrone for $\log t=8.35$ from Girardi et al. (2000) has been adjusted to $E(B-V)=0.38$ and $V-M_{V}=11.25$. The dashed curve is the same isochrone shifted by $0.75 \mathrm{mag}$ to reproduce the upper binary ridge. Filled and open circles have the same meaning as in Fig. 3.

with the same temperature and surface gravity, but not with the same $C_{0}(42-45)$ and $C_{0}(45-48)$ indices as the star in question. The successive columns of Table 6 show the star identification, the standard $D D O$ colours defined and derived as described by $\mathrm{PCM}$, the resulting $\triangle C N$ values, the number of iterations performed until the $[\mathrm{Fe} / \mathrm{H}]$ values converged within $0.01 \mathrm{dex}$ and the membership status assigned to each star. No $\triangle C N$ values could be determined for stars 96, 253 and 255 of NGC 6192 because they fall either outside the range of Janes (1977) Table 7 or outside the PCM calibration. The mean cyanogen anomalies for the confirmed cluster giants are: $\langle\Delta C N\rangle=0.120 \pm$ $0.016\left(\sigma_{p}\right), 0.030 \pm 0.015\left(\sigma_{p}\right)$ and $0.100 \pm 0.017\left(\sigma_{p}\right)$, for NGC 6192, NGC 6208 and NGC 6268, respectively, where $\sigma_{p}$ is the standard deviation of the mean. The cluster metallicities derived from the $[\mathrm{Fe} / \mathrm{H}]$ versus $\triangle C N$ relation given by $\mathrm{PCM}$ are: $[\mathrm{Fe} / \mathrm{H}]=+0.29 \pm 0.06,-0.05 \pm 0.05$ and $+0.22 \pm 0.06$, respectively. Therefore, NGC 6192 and NGC 6268 lie in the metalrich side of the metallicity distribution of open clusters, while NGC 6208 is found to be of nearly solar metal content.

The metallicity determined for NGC 6192, i.e., $[\mathrm{Fe} / \mathrm{H}]=$ +0.29 , does not agree with the value recently obtained by Paunzen et al. (2003), i.e. $[\mathrm{Fe} / \mathrm{H}]=-0.10$, based on uvby Strömgren's photometry. The latter, however, was determined without previously proving the membership status of the stars used to reach that conclusion. Thus, it is not possible to disregard the fact that such a result can have been influenced by the inclusion of non members of the cluster. As observable in Table 6, all CORAVEL non members of NGC 6192 have negative $\triangle C N$ values, which in turn imply negative values for the iron-to-hydrogen ratios. The marked differences between the metallicities of the field stars and those of NGC 6192 make the present metallicity determination more reliable.

The metallicities resulting from the $D D O$ analysis usually show a good agreement with spectroscopic determinations. However, for NGC 6192 and NGC 6268, the above solar values deduced from the photometric analysis does not permit us to 
Table 6. Standard $D D O$ colours and cyanogen anomalies.

\begin{tabular}{rcccrll}
\hline \hline Star & $C_{\mathrm{s}}(45-48)$ & $C_{\mathrm{s}}(42-45)$ & $C_{\mathrm{s}}(41-43)$ & $\Delta C N$ & $I$ & $\mathrm{Mem}$ \\
\hline NGC 192 & & & & & & \\
4 & 1.208 & 0.917 & 0.229 & -0.014 & 2 & $\mathrm{NM}$ \\
9 & 1.099 & 0.726 & 0.081 & 0.172 & 3 & $\mathrm{M}$ \\
28 & 1.384 & 1.125 & 0.328 & -0.062 & 2 & $\mathrm{NM}$ \\
45 & 1.150 & 0.734 & 0.167 & 0.100 & 2 & $\mathrm{M}$ \\
91 & 1.197 & 0.813 & 0.233 & 0.085 & 3 & $\mathrm{M}$ \\
137 & 1.223 & 0.853 & 0.264 & 0.122 & 4 & $\mathrm{M}$ \\
265 & 1.162 & 0.968 & 0.136 & -0.018 & 4 & $\mathrm{NM}$ \\
274 & 1.240 & 0.955 & 0.267 & -0.037 & 2 & $\mathrm{NM}$ \\
NGC 6208 & & & & & & \\
19 & 1.165 & 0.853 & 0.167 & 0.036 & 3 & $\mathrm{M}$ \\
27 & 1.115 & 0.752 & 0.113 & 0.032 & 2 & $\mathrm{NM}$ \\
31 & 1.151 & 0.791 & 0.162 & -0.004 & 3 & $\mathrm{M}$ \\
48 & 1.140 & 0.858 & 0.120 & 0.058 & 4 & $\mathrm{M}$ \\
133 & 1.185 & 0.913 & 0.190 & 0.102 & 3 & $\mathrm{NM}$ \\
$\mathrm{NGC} \mathrm{6268}$ & & & & & & \\
13 & 1.080 & 0.746 & 0.055 & 0.148 & 4 & $\mathrm{NM}$ \\
22 & 1.094 & 0.727 & 0.082 & 0.186 & 3 & $\mathrm{NM}$ \\
44 & 1.162 & 0.788 & 0.182 & 0.124 & 3 & $\mathrm{M}$ \\
48 & 1.241 & 0.913 & 0.276 & -0.051 & 3 & $\mathrm{NM}$ \\
73 & 1.180 & 0.822 & 0.203 & 0.120 & 2 & $\mathrm{NM}$ \\
76 & 1.136 & 0.770 & 0.142 & 0.076 & 2 & $\mathrm{M}$ \\
\hline
\end{tabular}

obtain satisfactory fits to the whole CMDs, especially in the red giant region. Isochrones with solar metallicity better reproduce the observations.

\section{Open clusters aligned along the line-of-sight of NGC 6192}

The derived metallicities for the three inner disk open clusters studied here seem to be compatible with the widely recognized existence of a radial abundance gradient in the Galactic disk (see, e.g. Chen et al. 2003), the inner regions being metal richer than the outer ones.

To examine the properties of a sample of inner disk open clusters located nearly in the same direction as the three clusters studied, we first searched the WEBDA Open Cluster Database (Mermilliod \& Paunzen 2003) for clusters with well determined $E(B-V)$ colour excesses and distances from the Sun. We limited the search to objects located at $(l, b)_{\mathrm{NGC} 6192} \pm 10^{\circ}$. When the $E(B-V)$ colour excesses and distances from the Sun were not provided by WEBDA, we used either the recent catalogue of Kharchenko et al. (2005) or appealed to the recent literature available. The catalogue of Kharchenko et al. (2005) is a result of studies of wide neighbourhoods of 513 open clusters and of 7 compact associations registered in a high precision homogeneous all sky catalogue ASCC-2.5 (Kharchenko 2001). We found a sample of 42 open clusters within the desired $(l, b)$ range that have well-determined fundamental parameters. In Table 7 we present the final cluster list (ranked according to increasing Galactic longitudes) with the values of the adopted parameters.

The distribution of the selected clusters in the $(l, b)$ plane is shown in Fig. 6. Those clusters are represented by open circles, whereas NGC 6192, NGC 6208 and NGC 6268 are depicted with filled circles. Most of the clusters projected towards the considered direction lie close to the Galactic plane, within $\pm 3^{\circ}$. Their distribution in the Galactic $(X, Y)$ plane is illustrated in Fig. 7. In addition, the Carina spiral arm and the line-of-sight from the Sun to NGC 6192 are represented by a solid line and by a straight line, respectively. Note that the distance between
Table 7. Fundamental parameters of selected open clusters.

\begin{tabular}{|c|c|c|c|c|c|}
\hline Cluster & $\begin{array}{c}l \\
\left(^{\circ}\right)\end{array}$ & $\begin{array}{l}b \\
\left({ }^{\circ}\right)\end{array}$ & $\begin{array}{c}E(B-V) \\
(\mathrm{mag})\end{array}$ & $\begin{array}{c}d \\
(\mathrm{kpc})\end{array}$ & Ref. \\
\hline Ruprecht 115 & 330.96 & -0.85 & 0.65 & 2.16 & 1 \\
\hline Pismis 22 & 331.47 & -0.60 & 2.00 & 1.00 & 1 \\
\hline Ruprecht 116 & 332.39 & -1.68 & 0.10 & 0.90 & 4 \\
\hline Ruprecht 118 & 332.55 & -1.80 & 0.41 & 0.34 & 1 \\
\hline NGC 6152 & 332.92 & -3.17 & 0.20 & 1.03 & 4 \\
\hline Ruprecht 119 & 333.28 & -1.88 & 0.57 & 0.96 & 1 \\
\hline NGC 6208 & 333.78 & -5.77 & 0.31 & 0.94 & 1,6 \\
\hline ESO 139-13 & 333.98 & -13.81 & 0.20 & 1.50 & 4 \\
\hline Pismis 23 & 334.67 & 0.43 & 2.00 & 2.60 & 1 \\
\hline NGC 6134 & 334.92 & -0.20 & 0.40 & 0.91 & 1 \\
\hline NGC 6167 & 335.22 & -1.43 & 0.78 & 1.11 & 1 \\
\hline NGC 6253 & 335.46 & -6.25 & 0.20 & 1.51 & 1 \\
\hline Ruprecht 120 & 336.39 & -0.49 & 0.70 & 2.00 & 1 \\
\hline NGC 6193 & 336.71 & -1.57 & 0.48 & 1.16 & 1 \\
\hline NGC 6200 & 338.00 & -1.07 & 0.58 & 2.05 & 1 \\
\hline Lynga 11 & 338.18 & 0.45 & 0.70 & 2.30 & 2 \\
\hline NGC 6178 & 338.41 & 1.21 & 0.22 & 1.01 & 1 \\
\hline NGC 6204 & 338.56 & -1.04 & 0.43 & 1.09 & 1 \\
\hline Hogg 22 & 338.56 & -1.14 & 0.65 & 1.22 & 1 \\
\hline Westerlund 1 & 339.56 & -0.40 & 3.87 & 5.50 & 5 \\
\hline IC 4651 & 340.09 & -7.91 & 0.12 & 0.89 & 1 \\
\hline NGC 6192 & 340.65 & 2.12 & 0.64 & 1.55 & 1 \\
\hline NGC 6216 & 340.67 & 0.01 & 0.45 & 4.30 & 1 \\
\hline NGC 6250 & 340.68 & -1.92 & 0.35 & 0.87 & 1 \\
\hline NGC 6124 & 340.74 & 6.02 & 0.75 & 0.51 & 1 \\
\hline Lynga 14 & 340.92 & -1.09 & 1.43 & 0.88 & 1 \\
\hline NGC 6249 & 341.54 & -1.19 & 0.44 & 0.98 & 1 \\
\hline NGC 6259 & 342.00 & -1.52 & 0.50 & 1.03 & 1 \\
\hline NGC 6231 & 343.46 & 1.18 & 0.44 & 1.24 & 1 \\
\hline Alessi 9 & 344.16 & -8.83 & 0.07 & 0.20 & 1 \\
\hline ESO $332-08$ & 344.40 & 1.81 & 0.20 & 1.20 & 4 \\
\hline ВН 205 & 344.60 & -1.62 & 0.30 & 2.16 & 4 \\
\hline Trumpler 24 & 344.70 & 1.50 & 0.42 & 1.14 & 1 \\
\hline NGC 6322 & 345.28 & -3.06 & 0.59 & 1.00 & 1 \\
\hline NGC 6242 & 345.47 & 2.47 & 0.38 & 1.13 & 1 \\
\hline NGC 6268 & 346.05 & 1.30 & 0.39 & 1.03 & 1 \\
\hline NGC 6281 & 347.73 & 1.97 & 0.15 & 0.48 & 1 \\
\hline NGC 6318 & 347.90 & -0.69 & 1.20 & 2.10 & 3 \\
\hline Ruprecht 125 & 348.50 & -3.40 & 0.10 & 1.50 & 4 \\
\hline Havlen-Moffat 1 & 348.70 & -0.77 & 1.85 & 2.90 & 1 \\
\hline BH 222 & 349.13 & -0.44 & 1.85 & 6.00 & 1 \\
\hline Trumpler 29 & 349.99 & -5.15 & 0.19 & 0.76 & 4 \\
\hline
\end{tabular}

References: (1) WEBDA (Mermilliod 2005); (2) Piatti et al. (2006); (3) Piatti et al. (2005); (4) Kharchenko et al. (2005); (5) Clark et al. (2005); (6) this work.

the outermost and the innermost clusters is more than $5 \mathrm{kpc}$ although their directions are close to that of the Galactic center. NGC 6192 is located behind the Carina spiral arm as seen from the Sun, while NGC 6208 and NGC 6268 are placed in front of this feature. Figure 8 was built taking advantage of this relatively long baseline in distance along this line of view, to examine the behaviour of the interstellar extinction along the direction considered. This figure shows the relationship between the visual interstellar absorption $A_{V}(=3.0 E(B-V))$ and the distance $d$ from the Sun. For comparison, we represented with a solid line the relationship for the Baade's window $\left[(l, b)=\left(1^{\circ},-3.9^{\circ}\right)\right]$ obtained by $\mathrm{Ng}$ et al. (1996). A close look at this figure revealed the following features: (1) BH 222 and Westerlund 1 are the farthest open clusters of the selected sample. They are located at $\sim 6 \mathrm{kpc}$ (Piatti \& Clariá 2002) and $\sim 5.5 \mathrm{kpc}$ (Clark et al. 2005) from the Sun and at $\sim 2.8 \mathrm{kpc}$ and $\sim 3.2 \mathrm{kpc}$ from the Galactic center, respectively. (2) At the distance of these two clusters from the 


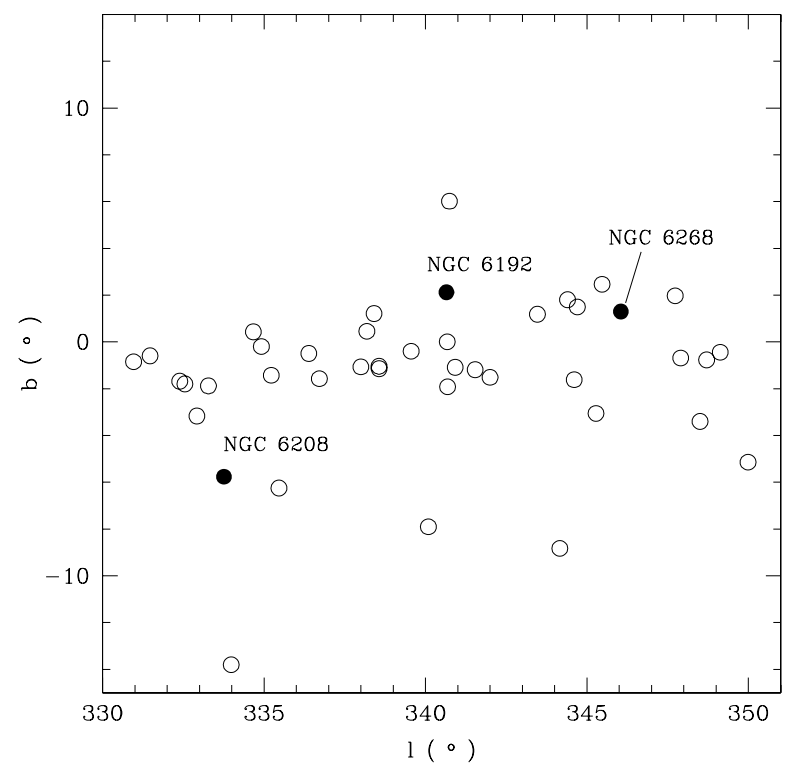

Fig. 6. Galactic coordinates $(l, b)$ of inner disk open clusters located in the direction of NGC 6192 is.

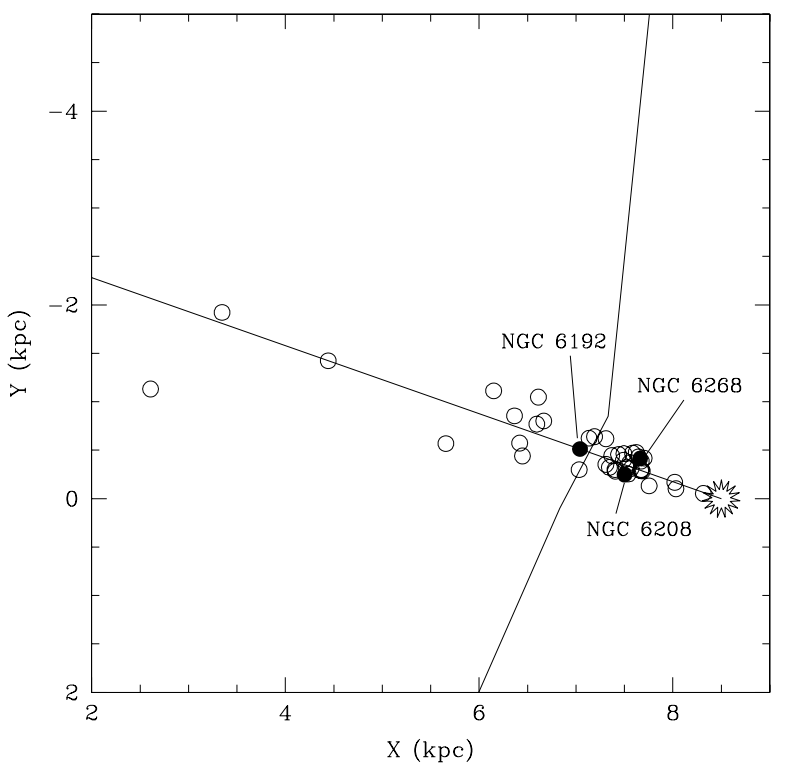

Fig. 7. The Galactic plane with the $(X, Y)$ positions of the open clusters located in the direction of NGC 6192. The Carina spiral arm (solid line), the line-of-sight from the Sun to NGC 6192 (straight line) and the position of the Sun are indicated.

Sun, the visual interstellar absorption towards the Baade's window - not far from the direction of NGC 6192 - is between 4 and 9 mag smaller. It is surprising that, in spite of being located at approximately the same distance, these two clusters appear to be affected by such different visual absorptions. Although significant differential reddening across the field of Westerlund 1 has been recently confirmed by Clark et al. (2005) and according to the same authors its distance of $5.5 \mathrm{kpc}$ represents only an upper limit, the difference of $\sim 5$ mag in their visual absorptions still appears to be too high. The reddening of Westerlund $1-$ the most massive compact young cluster identified in the Local Group up to now (Clark et al. 2005) - is produced in front of the Carina spiral arm, probably caused by a single small dark cloud (Piatti et al. 1998). (3) NGC 6216, located farther than $4 \mathrm{kpc}$ from the Sun, is reddened as expected if it were situated exactly in the

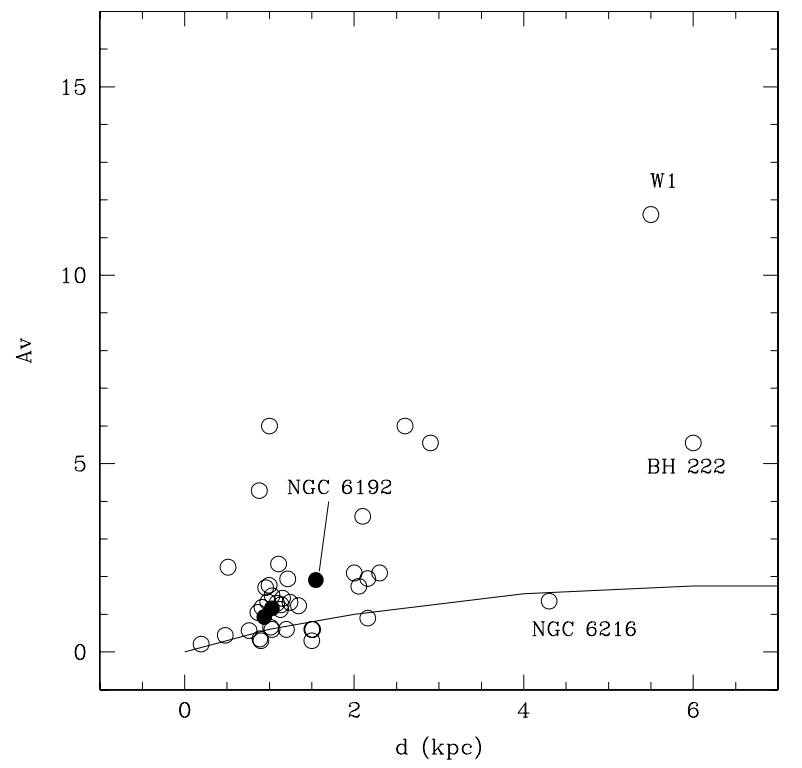

Fig. 8. Visual interstellar absorption $A_{V}$ vs. distance $d$ from the Sun. The relationship corresponding to Baade's window is indicated.

Baade's window direction. (4) Although most of the clusters located closer than $2 \mathrm{kpc}$ from the Sun are usually more reddened than what would correspond to the Baade's window absorption law, they are close to it.

\section{Summary and conclusions}

New $U B V$ amd $D D O$ photometry and CORAVEL radial-velocity data of a sample of red giant candidates in the fields of the inner disk open clusters NGC 6192, NGC 6208 and NGC 6268 have allowed us to determine the membership of red giants in these three clusters. Three new spectroscopic binaries have been discovered among the red giants of NGC 6192 and NGC 6208. Mean radial velocities and $E(B-V)$ colour excesses of $(-7.72 \pm 0.38) \mathrm{km} \mathrm{s}^{-1},(-32.21 \pm 0.28) \mathrm{km} \mathrm{s}^{-1}$ and $(-15.11 \pm 0.08) \mathrm{km} \mathrm{s}^{-1}$ and $0.63 \pm 0.03,0.31 \pm 0.02$ and $0.44 \pm 0.03$ mag were derived for NGC 6192, NGC 6208 and NGC 6268, respectively.

NGC 6192 is a good example of how different estimates of the interstellar reddening in the field of an open cluster influence the derived cluster parameters. Previous estimates of the cluster $E(B-V)$ colour excess vary between 0.26 and 0.68 mag, which imply very discrepant distances and ages. The reddening here derived for NGC 6192, i.e., $E(B-V)=0.63$, supports the cluster parameters derived by KF91. On the other hand, NGC 6208 is found to be $\sim 0.1$ mag more reddened than was previously believed, while the reddening derived here for NGC 6268 shows good agreement with the one quoted in the literature.

$D D O$ abundance indices yield mean cluster metallicities of $[\mathrm{Fe} / \mathrm{H}]=+0.29 \pm 0.06,-0.05 \pm 0.05$ and $+0.22 \pm 0.06$ for NGC 6192, NGC 6208 and NGC 6268, respectively, which appear to be compatible with the existence of a radial abundance gradient in the Galactic disk. An inspection of the properties of 42 known open clusters nearly aligned along the line-of-sight of NGC 6192 as seen from the Sun shows that BH222 and Westerlund 1 are the farthest ones, their absorptions being between 4 and 9 mag larger than the values that would correspond if these clusters were located exactly in the direction of Baade's window. Evidence is presented that most of the clusters located closer than $2 \mathrm{kpc}$ from the Sun are slightly more 
reddened than the absorption resulting from the Baade's window absorption law.

Acknowledgements. We are grateful to the La Silla and CTIO staff members and night assistants for their kind hospitality and support during the observing runs. This research was partially supported by the Argentinian Institutions CONICET, SECYT (Universidad Nacional de Córdoba), Agencia Nacional de Promoción Científica y Tecnológica (ANPCyT) and Agencia Córdoba Ciencia. This work is based on observations made at CTIO, which is operated by AURA Inc., under cooperative agreement with the NSF.

\section{References}

Archinal, B. A., \& Hynes, S. J. 2003, Star Clusters (Willmann-Bell, Inc.) Baranne, A., Mayor, M., \& Poncet, J.-L. 1979, Vistas in Astron., 23, 279 Baranne, A., Queloz, D., Mayor, M., et al. 1996, A\&AS, 119, 373 Chen, L., Hou, J. L., \& Wang, J. J. 2003, AJ, 125, 1397

Claret, A., Paunzen, E., \& Maitzen, H. M. 2003, A\&A, 412, 91 Clariá, J. J. 1985, A\&AS, 59, 195

Clariá, J. J., \& Lapasset, E. 1983, J. Astrophys. Astron., 4, 117

Clariá, J. J., Piatti, A. E., \& Lapasset, E. 1994, PASP, 106, 436

Clariá, J. J., Piatti, A. E., Lapasset, E., \& Mermilliod, J.-C. 2003, A\&A, 399, 543 Clariá, J. J., Piatti, A. E., Lapasset, E., \& Parisi, M. C. 2005, Baltic Astron., 14, 301

Clark, J. S., Negueruela, I., Crowther, P. A., \& Goodwin, P. 2005, A\&A, 434, 949

Collinder, P. 1931, Medd. Lunds. Astron. Obs., 2

Cousins, A. W. J. 1973, Mem. R. Astron. Soc., 77, 223

Cousins, A. W. J. 1974, Mon. Not. Astron. Soc. Sth. Africa, 33, 149

Crawford, D. L. 1978, AJ, 83, 48

Girardi, L., Bressan, A., Bertelli, G., \& Chiosi, C. 2000, A\&AS, 141, 371

Graham, J. A. 1982, PASP, 92, 244

Janes, K. A. 1977, PASP, 89, 576

Kilambi, G. C., \& Fitzgerald, M. P. 1983, Bull. Astron. Soc. India, 11, 226 (KF83)

Kharchenko, N. V. 2001, Kinematika I Fizika Nebesnykh Tel., 17(5), 409

Kharchenko, N. V., Piskunov, A. E., Roeser, S., Schilbach, E., \& Scholz, R. D. 2005, A\&A, 438, 1163
King, D. J. 1987, The Observatory, 107, 107

Kjeldsen, H., \& Frandsen, S. 1991, A\&AS, 87, 119 (KF91)

Lindoff, U. 1972, A\&AS, 7, 231

Loktin, A., Gerasimenko, T., \& Malisheva, L. 2001, A\&A Trans., 20, 605

Loktin, A., \& Matkin, N. V. 1994, A\&A Trans., 4, 153

McClure, R. D. 1973, Spectral Classification and Multicolour Photometry (Dordrecht, Holland: D. Reidel Publishing Co.), 192 McClure, R. D. 1976, AJ, 81, 182

McSwain, M. V., \& Gies, D. R. 2005, A\&A, 161, 118

Melotte, P. J. 1915, Mem. R. Astron. Soc., 60, 175

Mermilliod, J.-C., Clariá, J. J., Andersen, J., \& Mayor, M. 1997, A\&A, 324, 91

Mermilliod, J.-C., Clariá, J. J., Andersen, J., Piatti, A. E., \& Mayor, M. 2001, A\&A, 375, 30

Mermilliod, J.-C., \& Paunzen, E. 2003, A\&A, 410, 511

Ng, Y. K., Bertelli, G., Chiosi, C., \& Bressan, A. 1996, A\&A, 310, 771

Paunzen, E. 2006, private communication

Paunzen, E., \& Maitzen, J. M. 2001, A\&A, 373, 153

Paunzen, E., Maitzen, J. M., Rakos, K. D., \& Schombert, J. 2003, A\&A, 403, 937

Paunzen, E., Netopil, M., Iliev, Ikh., et al. 2005, A\&A, 443, 157

Perryman, M. A. C., Brown, A. G. A., Lebreton, Y., et al. 1998, A\&A, 331, 81

Piatti, A. E., \& Clariá, J. J. 2002, A\&A, 388, 179

Piatti, A. E., Clariá, J. J., \& Minnniti, D. 1993, J. Astrophys. Astron., 14, 145 (PCM)

Piatti, A. E., Clariá, J. J., \& Abadi, M. G. 1995, AJ, 110, 2813

Piatti, A. E., Bica, E., \& Clariá, J. J. 1998, A\&AS, 127, 423

Piatti, A. E., Clariá, J. J., \& Ahumada, A. V. 2005, PASP, 117, 22

Piatti, A. E., Clariá, J. J., \& Ahumada, A. V. 2006, New Astron., 11, 262

Seggewiss, W. 1967, Thesis, Univ. Munster

Seggewiss, W. 1968, Z. Astrophys., 68, 142

Straiz̃ys, V. 1992, Multicolour Stellar Photometry (Tucson, Arizona: Pachart Publishing House)

Trumpler, R. J. 1930, Lick Obs. Bull., 14, 154

Twarog, B. A., Ashman, K. M., \& Anthony-Twarog, B. J. 1997, AJ, 114, 2556

Udry, S., Mayor, M., \& Queloz, D. 1999, in Precise stellar radial velocities, ed. J. B. Hearnshaw, \& C. D. Scarfe, IAU Colloq., 170, ASP Conf. Ser., 185, 367

van den Bergh, S., \& Hagen, G. L. H. 1975, AJ, 80, 11 